\title{
Assessment of Reliability and Validity of the Mental Chronometry Task Based on the Box and Block Test in Multiple Sclerosis Patients
}

\author{
Maryam Rezaeian ${ }^{1}$, Zahra Assadollahi², Hossien Azinn ${ }^{3,4}$, Ayat Kaeidi'5,6 and Mahdieh Azin ${ }^{5,6, *}$ \\ 1 Department of Neuroscience, Faculty of Advanced Technologies in Medical Sciences, Iran University of Medical Sciences, Tehran, Iran \\ 2 Department of Epidemiology and Biostatistics, Occupational Environmental Research Center, Rafsanjan University of Medical Sciences, Rafsanjan, Iran \\ ${ }^{3}$ Noncommunicable Diseases Research Center, Rafsanjan University of Medical Sciences, Rafsanjan, Iran \\ ${ }_{4}$ Department of Neurology, School of Medicine, Rafsanjan University of Medical Sciences, Rafsanjan, Iran \\ ${ }^{5}$ Physiology-Pharmacology Department, School of Medicine, Rafsanjan University of Medical Sciences, Rafsanjan, Iran \\ ${ }^{6}$ Physiology-Pharmacology Research Center, Research Institute of Basic Medical Sciences, Rafsanjan University of Medical Sciences, Rafsanjan, Iran
}

* Corresponding author: Mahdieh Azin, Physiology-Pharmacology Department, School of Medicine, Rafsanjan University of Medical Sciences, Rafsanjan, Iran, Physiology-Pharmacology Research Center, Research Institute of Basic Medical Sciences, Rafsanjan University of Medical Sciences, Rafsanjan, Iran. Tel: 00983431315079, +989132908035; Email: mahdieh.azin@gamil.com

Received 2020 December 29; Revised 2021 February 13; Accepted 2021 February 25.

\begin{abstract}
Background: Motor imagery (MI) is the visualization of action without its overt performance. One of the measures of explicit MI is mental chronometry which has been applied to multiple sclerosis (MS) patients; nonetheless, thereliability and validity of this tool has been never confirmed.

Objectives: Therefore, the present study aimed to assess the reliability and validity of mental chronometry in MS patients.

Methods: A number of 60 MS patients who met the inclusion criteria were included in the present study via the census method Thereafter, 20 MS patients were tested via mental chronometry based on the box and block test, as well as kinesthetic and visual imagery questionnaire-20 (KVIQ-20) in two sessions with a 10-day interval. Intra-class correlation coefficients (ICCs) were calculated to determine the test-retest reliability of mental chronometry. Pearson's correlation analysis was used to evaluate criterion validity with the KVIQ-20.

Results: The test-retest reliability for the mental chronometry was good (ICCs: visual analogue scale $=0.88$, mean execution and motor imagery absolute difference $=0.75$, imagery duration $=0.91$, and execution duration $=0.97$ ). Moreover, the concurrent validity between the visual analogue scale of mental chronometry and KVIQ-20 was good.

Conclusion: As evidenced by the results of the present study, the mental chronometry based on box and block is a reliable and valid tool for the assessment of motor imagery in MS patients.
\end{abstract}

Keywords: Mental chronometry, Motor imagery, Multiple sclerosis, Test-retest reliability

\section{Background}

Multiple sclerosis (MS) is one of the most common autoimmune and demyelinating diseases of the central nervous system (1). Neural lesions can lead to sensory and motor dysfunction, as well as cognitive impairment (2). The underlying cause of MS is still unknown, and numerous genes moderately increase disease susceptibility. In addition, several environmental factors, such as vitamin D deficiency, exposure to UV radiation, Epstein-Barr virus (EBV) infection, obesity, and smoking, can increase this susceptibility (3).

Cognitive impairment is present in $45 \%-60 \%$ of MS patients and can occur in the early stages of the disease (4). MS is usually diagnosed during the years of employment and productivity, and cognitive impairment has a severe impact on behavior and social functioning, daily life activities, and patients' employment $(5,6)$. It affects not only patients but also their family relationships; moreover, it is the reason behind frequent complaints from caregivers (6). Language, learning and memory, complex attention, executive function, perceptual-motor function, and social cognition are among the cognitive functions (7) which are impaired in MS patients (8). Cognitive impairment can be due to inflammatory and neurodegenerative changes in the white and gray matter of the brain on both global and local scales (9).

One of the cognitive functions is Motor imagery (MI) which is defined as follows: MI is the mental ability to imagine a specific movement without performing any overt movement (10). It is a cognitive process of movement which represents preparing, decision making, and planning movement involving such brain regions as the supplementary motor area, premotor area, primary sensorimotor area, posterior parietal lobe, striatum, cerebellum, and thalamus (11).

The two aspects of motor imagery are referred to as explicit and implicit (12). Explicit motor imagery is defined as the conscious imagining of an action. This ability is assessed by a mental chronometry test and motor imagery questionnaires (13). In the implicit motor imagery, some tests, such as mental rotation, are applied to enable individuals to use motor imagery ability unconsciously (12). Mental chronometry indicates a temporal difference of imagery and executed movements with the vividness 
of the imagined movement (14).

Motor imagery questionnaires evaluate the vividness of motor imagery (15), and mental rotation provides data about the reaction time and accuracy rate of laterality judgments of body images (16). Each test that is used in motor imagery examines one aspect of imaging; therefore, it is necessary to test the ability of motor imagery from various aspects before the administration of mental training based on motor imagery.

Motor imagery is impaired in MS patients as demonstrated by their reaction time and correct response number in the hand mental rotation task $(16,17)$. Their scores of mental chronometry, as well as kinesthetic and visual imagery questionnaire-20 (KVIQ-20) were compared to those of healthy subjects $(14,18)$. KVIQ-20 is a sale designed for the assessment of visual and kinesthetic motor imagery ability. Since the reliability and validity of KVIQ-20 has been confirmed in MS patients, this questionnaire was used in the current study (15).

Mental chronometry based on the box and block test compares the duration of imagery and physical execution of a task. A close temporal relationship represents correct motor imagery (14). Mental chronometry has been reported to have acceptable test-retest reliability and internal consistency in stroke patients (19). Due to the multifaceted nature of motor imagery, it is necessary to examine in detail various tests of motor imagery ability (20). In the past three decades, the field of cognitive impairment in MS has dramatically improved. This knowledge encompasses the preparation of sensitive neuropsychological battery tests for clinical research, and a better understanding of defects in the neural correlates of cognitive impairment with the help of neuroimaging and treatment strategies, rehabilitation, and prevention.

Cognitive function evaluation should be included in the standard clinical evaluation of MS patients. Limitations in the study of cognitive impairment still exist due to the validation and standardization of both diagnostic and therapeutic tools (21). The validity and reliability of mental chronometry based on the box and block test have not been investigated in MS patients.

\section{Objectives}

The present study aimed to examine the reliability and validity of mental chronometry based on the box and block test in MS patients.

\section{Methods}

Regarding ethical consideration, the study protocols were approved by the Ethics Committee of Rafsanjan University of Medical Sciences and adhered to the principles of the Helsinki Declaration
(IR.RUMS.REC.1397.238). In 2018, 60 relapseremitting MS patients were selected via the census method from Rafsanjan and Kerman, Iran.

In this cross-sectional study, the participants were MS patients selected from private neurological clinics in Kerman and governmental neurological clinics in Rafsanjan. Over a period of approximately 10 months, 60 MS patients who met the inclusion criteria and were willing to cooperate were enrolled in the study. According to a similar previous study (15), 20 patients performed tasks again in order to test-retest measurement. Another study was performed on 14 Parkinson's patients with the aim of validating the mental chronometry task (22). An expert administered the tests to the patients in a neurological clinic. The inclusion criteria were as follows:

1- The subjects were diagnosed with MS through neurological examinations and analysis of their medical documents by a neurologist based on the revised McDonald criteria (23).

2- The subjects had relapsing-remitting MS according to the National Multiple Sclerosis Society.

3- They were right-handed based on the Edinburg Handedness Inventory Questionnaire (24).

4-They had no relapsing MS for at least three months before the study.

5 - They did not receive any psychotherapy.

6- Their scores of mental status according to the mini-mental state examination were higher than 24.

7. Their EDSS scores were lower than 3.5.

8- Their MMSE scores were more than 24.

9- The subjects signed a written consent form.

Their demographic data, expanded disability status scale, and mini-mental state examination were recorded. Thereafter, the subjects' motor imagery was evaluated via KVIQ-20. The subjects were also asked to perform the box and block test which provides information about the mental chronometry of explicit imagery. The patients' disability rate was determined by a neurologist using an expanded disability status scale (25). This scale was utilized to assess physical disability rate and functional systems in MS patients who had a score ranging from 0-10 with half-point increments. Mini-Mental State Examination (MMSE) was conducted on the patients (26). This 30-point test assesses orientation, registration, attention, calculation, recall, copying, and language. A score of one is assigned to each correct answer.

\subsection{Kinesthetic and visual imagery questionnaire}

Kinesthetic and visual imagery refers to visualized movements without performing any obvious and real movements and is examined by KVIQ-20. The reliability and validity of this questionnaire were confirmed for MS patients in 2013 (15). In the present study, this questionnaire was used as a standard scale to evaluate the 
criterion validity of the mental chronometry based box and block. This questionnaire aims to determine the ability to visualize movements and the extent to which those imagined movements are felt. The questionnaire was completed by the examiner. All the movements were examined while the subject was sitting. The 20 -item questionnaire is composed of a visual imagery scale and a kinesthetic imagery scale. The items included 1- neck flexion/extension, 2 -shoulder shrugging, 3-forward shoulder flexion on the non-dominant side, 4- elbow flexion on the dominant side, 5- thumb to fingertips on the dominant side, 6- forward trunk flexion, 7- knee extension on the non-dominant side, 8- hip abduction on the dominant side, 9- foot tapping on the non-dominant side, as well as 10-foot external rotation on the dominant side. Items 1, 2 and 6 were considered axial subsets. Items 3,4 and 5 were regarded as upper limbs. The lower limb subsets include items 7-10.

While sitting, the patient was asked to perform a movement, which was first executed by the examiner; thereafter, he/she should visualize the same movement. Here, the patient did not perform the movement but only imagined movement he performed in the previous stage. Finally, the patient was asked to clearly state the image in his mind and the intensity of sensation. Based on patient's description, a score from 1-5 was allocated by the examiner (1: no image/no sensation; 5: image as clear as is being seen /as intense as executing the action) (15). The lowest and highest score of each patient based on this questionnaire is 20 and 100 , respectively.

\subsection{Mental chronometry based on Box and Block test}

The box and block test was used in MS patients in order to evaluate the mental chronometry of upper limbs. Mental chronometry is obtained based on the comparison of physical execution duration with motor imagery duration. If the duration of the execution task is similar to that of motor imagery task, the subject has fine motor imagery ability. Three trials of physical execution and three trials of imagery were performed on each hand, and the order of trials was selected randomly.

The patients were provided by the test box with a length of $53.7 \mathrm{~cm}$, which is divided into two parts by a board with a height of $15.2 \mathrm{~cm}$ (27). During this test, the patient was required to transfer 20 wooden blocks $\left(20 \mathrm{~cm}^{2}\right)$ once with the dominant hand, followed by imagining the same action with the same hand. Following that, the patient was asked to move the blocks with the non-dominant hand and was then asked to imagine doing the same movement with that hand. For each trial, the examiner recorded the task duration.

During the imagery trial, the patient uses first person visual imagery. The absolute value of time duration difference between the real movement and the motor imagery movement was calculated. Apart from recording the motor imagery duration, the examiner asked about the vividness of motor imagery using seven modes $(1=$ very easy imagery to $7=$ very difficult imagery) (14). The four variables of mental chronometry include the execution duration, motor imagery duration, absolute difference of execution and motor imagery, and visual analogue scale. In the second session, which was held after 10 days, KVIQ20 and mental chronometry were repeated exactly in the same way as before.

\subsection{Statistical analyses}

Test-Retest Reliability with a 10-Day interval was used to measure the reliability of mental chronometry test. Execution duration, motor imagery duration, execution and motor imagery absolute difference, as well as visual analogue scale, were the variables of mental chronometry. Cronbach's alpha was calculated to assess intra-class correlation coefficient (ICC). The ICCs within 0.90-0.99 are considered to indicate high reliability levels, those between 0.80 and 0.89 signify good reliability levels, those between 0.70 and 0.79 denote fair reliability levels, and those below 0.69 indicate poor reliability levels.

In order to evaluate the criterion validity of mental chronometric task, Pearson correlation test was used between variable of mental chronometry and those of KVIQ-20 as a standard questionnaire. Correlation coefficient of significance is regarded as adequate evidence of validity. The skewness index of all variables was in the range of $-3-+3$; consequently, the assumption of normal frequency distribution of all variables was accepted. All statistical assessments were two-tailed, and a p-value less than 0.05 was considered statistically significant. All statistical analyses were carried out in SPSS software (version 21, Chicago, IL, USA).

\section{Results}

A number of 60 MS patients $(20$ males and 40 females) were included in the current study. Table 1 displays the demographic characteristics of patients. The mean age scores of patients and the mean duration of disease were obtained at $30.5 \pm 7.2$ years ( $\min =20$ and $\max =44$ years) and $48.9 \pm 51.7$ months (min=6 months and $\max =276$ months), respectively (Table 1). Descriptive statistics of

\begin{tabular}{lc}
\hline Table 1. Subject demographic characteristics $(\mathrm{n}=60)$ \\
\hline Sex: Male (\%) & $20(33.3)$ \\
Sex: Female (\%) & $40(66.7)$ \\
Age (year, mean \pm SD) & $30.5 \pm 7.2$ \\
\hline Education (year, mean \pm SD) & $13.42 \pm 3.25$ \\
Duration of disease (month, mean \pm SD) & $48.90 \pm 51.7$ \\
Range of expanded disability status scale & $0.5-3.5$ \\
\hline Range of mini mental state examination & $25-30$ \\
\hline
\end{tabular}


Table 2. Descriptive statistics of mental chronometry task and KVIQ-20 test ( $\mathrm{n}=60$ )

\begin{tabular}{lcccc}
\hline Variables & Minimum & Maximum & Mean & Std. Deviation \\
\hline Mean absolute difference of execution and motor imagery (second) & .39 & 17.50 & 5.41 & 3.66 \\
Mean visual analogue scale of both hands & 2.33 & 7.00 & 4.7 & 1.42 \\
Total KVIQ-20 & 20.00 & 99.00 & 66.5 & 14.39 \\
\hline
\end{tabular}

variables of mental chronometry and KVIQ-20 are presented in Table 2 .

The time interval between the test and retest sessions was 10 days. The value of the intra-class correlation coefficient (ICC) and 95\% confidence interval (CI) for the mental chronometry test and KVIQ-20 are reported in Table 3. As indicated in Table 3, the value of Cronbach's alpha coefficients obtained in all aspects of the mental chronometry test and KVIQ-20 is appropriate and signifies the reliability of an acceptable instrument and questionnaire.

In order to determine the validity of mental chronometry test, a comparison of the score obtained from the KVIQ-20 and the visual analogue scale of the mental chronometry test is provided in Table 4 . The correlation between the variables of mental chronometry and KVIQ-20 was also explored. The overall score of KVIQ-20 was strongly correlated with total visual analogue scale $(\mathrm{r}=0.697 ; \mathrm{P}<0.001)$. Other correlations were also strong and significant. These results pointed out that the high validity of the visual analogue scale is related to mental chronometry test. The lowest and highest correlations of the visual analogue scale are respectively related to visual upper limb $(\mathrm{r}=0.550 ; \mathrm{P}<0.001)$ and kinesthetic upper limb $(\mathrm{r}=0.758 ; \mathrm{P}<0.001)$. Table 4 illustrates the correlation of the scores obtained from the KVIQ-20 and the variables of the mental chronometry test. KVIQ-20 score has a significant correlation with imagery trial of the mental chronometry test $(\mathrm{P}<0.05)$. Nonetheless, KVIQ-20 showed no significant correlation with the mean difference and execution trial of the mental chronometry test ( $P>0.05$; Table 4).

\begin{tabular}{|c|c|c|c|}
\hline Task & Variables & ICC (CI 95\%)* & P-value \\
\hline \multirow{4}{*}{$\begin{array}{l}\text { The mental } \\
\text { chronometry test }\end{array}$} & Visual analogue scale & $0.886(0.703-0.956)$ & $\mathrm{P}<0.001$ \\
\hline & Absolute difference of execution and motor imagery & $0.753(0.358-0.905)$ & 0.002 \\
\hline & Imagery duration & $0.915(0.779-0.967)$ & $\mathrm{P}<0.001$ \\
\hline & Execution duration & $0.971(0.925-0.989)$ & $\mathrm{P}<0.001$ \\
\hline \multirow{9}{*}{ KVIQ-20 } & Total KVIQ-20 & $0.980(0.950-0.992)$ & $\mathrm{P}<0.001$ \\
\hline & Visual subset & $0.9883(0.959-0.993)$ & $\mathrm{P}<0.001$ \\
\hline & Axial visual subset & $0.945(0.863-0.978)$ & $\mathrm{P}<0.001$ \\
\hline & upper limb visual subset & $0.988(0.971-0.995)$ & $\mathrm{P}<0.001$ \\
\hline & lower limb visual subset & $0.969(0.924-0.987)$ & $\mathrm{P}<0.001$ \\
\hline & Kinesthetic subset & $0.936(0.843-0.974)$ & $\mathrm{P}<0.001$ \\
\hline & axial kinesthetic subset & $0.902(0.759-0.960)$ & $\mathrm{P}<0.001$ \\
\hline & upper limb kinesthetic subset & $0.926(0.818-0.970)$ & $\mathrm{P}<0.001$ \\
\hline & lower limb kinesthetic subset & $0.936(0.843-0.974)$ & $\mathrm{P}<0.001$ \\
\hline
\end{tabular}

Table 4. Pearson correlation coefficients between the scores obtained from the KVIQ-20 and the visual analogue scale and imagery and execution of the mental chronometry test $(n=60)$

\begin{tabular}{|c|c|c|c|c|}
\hline Variables & $\begin{array}{c}\text { Mean Visual analogue scale } \\
\text { r (P-value) }\end{array}$ & $\begin{array}{l}\text { Mean difference } \\
\text { r (P-value) }\end{array}$ & $\begin{array}{c}\text { Mean imagery duration } \\
\text { r (P-value) }\end{array}$ & $\begin{array}{c}\text { Mean execution duration } \\
\text { r (P-value) }\end{array}$ \\
\hline Total KVIQ - 20 & $0.697(\mathrm{P}<0.001)$ & $-0.204(\mathrm{P}<0.117)$ & $-0.319(\mathrm{P}=0.013)$ & $-0.233(\mathrm{P}<0.074)$ \\
\hline Visual-subset & $0.587(\mathrm{P}<0.001)$ & $-0.098(\mathrm{P}=0.455)$ & $-0.335(\mathrm{P}=0.009)$ & $-0.209(\mathrm{P}=0.109)$ \\
\hline Visual axial & $0.582(\mathrm{P}<0.001)$ & $-0.109(\mathrm{P}=0.405)$ & $-0.381(\mathrm{P}=0.003)$ & $-0.286(\mathrm{P}=0.027)$ \\
\hline Visual upper limb & $0.550(\mathrm{P}<0.001)$ & $-0.136(\mathrm{P}=0.302)$ & $-0.291(\mathrm{P}=0.024)$ & $-0.193(\mathrm{P}=0.139)$ \\
\hline Visual lower limb & $0.553(\mathrm{P}<0.001)$ & $-0.048(\mathrm{P}=0.713)$ & $-0.298(\mathrm{P}=0.021)$ & $-0.139(\mathrm{P}=0.290)$ \\
\hline Kinesthetic-subset & $0.737(\mathrm{P}<0.001)$ & $-0.292(\mathrm{P}=0.024)$ & $-0.269(\mathrm{P}=0.038)$ & $-0.232(\mathrm{P}=0.074)$ \\
\hline Kinesthetic axial & $0.646(\mathrm{P}<0.001)$ & $-0.250(P=0.054)$ & $-0.288(\mathrm{P}=0.026)$ & $-0.242(\mathrm{P}=0.063)$ \\
\hline Kinesthetic upper limb & $0.758(\mathrm{P}<0.001)$ & $-0.327(\mathrm{P}=0.011)$ & $-0.215(\mathrm{P}=0.099)$ & $-0.241(\mathrm{P}=0.064)$ \\
\hline Kinesthetic lower limb & $0.713(\mathrm{P}<0.001)$ & $-0.266(\mathrm{P}=0.040)$ & $-0.270(\mathrm{P}=0.037)$ & $-0.197(\mathrm{P}=0.132)$ \\
\hline
\end{tabular}

\section{Discussion}

Mental training based on motor imagery has been recently considered a promising method of rehabilitation for neurodegenerative patients. In order to determine the effectiveness of this method in patients' rehabilitation, it is necessary to assess various dimensions of motor imagery abilities of patients using tasks, as well as valid and reliable questionnaires. Elke Heremans introduced the use of the box and block task for the assessment of mental chronometry in Parkinson's and MS patients $(14,22)$. To the best of our knowledge, the present study is the first to measure the reliability and validity of mental chronometry based on the box and block test in MS patients. In order to determine the reliability of this test, all variables of mental chronometry were analyzed in two sessions with a 10-day interval. 
The KVIQ-20 whose reliability and validity have been confirmed was used to check the validity of the mental chronometry. This scale is a valid test designed for the assessment of motor imagery ability in MS patients (15). The correlation between variables of KVIQ-20 and mental chronometry was calculated to examine the validity of the mental chronometry. The results of the present study indicated that mental chronometry based on the box and block test is a reliable instrument for assessing motor imagery ability in MS patients.

All ICC values for all variables of mental chronometry based on the box and block test for two sessions were 0.75 or higher, pointing to the acceptable stability of this task in MS patients over time. In line with the findings of the present study, a previous study conducted on stroke patients determined the reliability of mental chronometry using time-dependent motor imagery screening test and temporal congruence test (19). The highest and lowest ICCs of mental chronometry task belonged to the execution duration and absolute difference of execution and motor imagery, respectively. Regarding the ICC values, the reliability was higher when the patients executed the tasks, rather than when they imagined performing them.

The present study also evaluated the reliability of KVIQ-20 in MS patients. The results indicated high reliability level for the total KVIQ-20 score. A similar study has been conducted, and the results confirmed our finding (20). Furthermore, the content validity of the mental chronometry variables (the visual analogue scale, imagery and execution duration) and total KVIQ-20 score were obtained at $0.697,-0.32$ and -0.23 , respectively. In addition, total KVIQ-20 score was a significantly correlated with visual analogue scale and imagery duration. Nevertheless, no significant correlation was found between KVIQ-20 score and execution duration in the mental chronometry.

The results of the current study observed a strong positive correlation between the total score of KVIQ20 and total visual analogue scale, pointing to the convergent validity of the visual analogue scale of mental chronometry. This positive significant correlation was due to the fact that the patients achieved higher scores when the task was easy; however, when the task was difficult, they obtained lower scores. A significant correlation was detected between visual analogue scale and KIVQ-20 since both of them were related to the vividness of imagery, and their variables were comparable. Visual analogue scale was used for obtaining the validity of the mental chronometry test. Due to the good stability and validity of the visual analogue scale and its comparable results with KVIQ-20, visual analogue scale of mental chronometry can be used in MS patients.

Furthermore, our findings showed that the KVIQ-
20 score had a significant but negative correlation with imagery duration of the mental chronometry. Moreover, there was a negative but not significant correlation between KVIQ-20 score and the execution duration of mental chronometry. This negative correlation can be ascribed to more execution and imagery duration when the task was difficult for the patient. As expected, no significant correlation was observed between the duration of execution in the mental chronometry and KIVQ-20 variables which can be attributed to different nature of their variables.

Considering the limitations of the present study, it was the first to assess the reliability and validity of the mental chronometry test based on box and block test in MS patients; therefore, there was no valid mental chronometry test to be compared with the test used in this study. Furthermore, mental chronometry based on the box and block test was used to assess the motor imagery ability in the upper limb and not the lower limb.

\section{Conclusion}

As evidenced by the obtained results, mental chronometry based on the box and block test is a reliable and valid test for MS patients to determine whether the patient can participate in mental imagery training and benefit from motor imagery rehabilitation or not. In conclusion, in order to gain a better insight into patient's ability to engage in motor imagery training, the assessment of several motor imagery aspects seems necessary prior to the rehabilitation program.

\section{Acknowledgments}

This research project was financially supported by a grant from Rafsanjan University of Medical Sciences.

\section{Footnotes}

Authors' Contribution: Mahdieh Azin, Hossien Azin and Ayat Kaeidi devised the project and the main conceptual ideas. Hossien Azin and Maryam Rezaeian performed the experiments. Zahra Assadollahi analyzed and interpreted the result. All authors prepared the first draft of the manuscript. All authors have read and approved the final manuscript.

Conflict of Interests: All the authors declared that there are no personal, financial, commercial, or academic conflict of interest.

Ethical Approval: The study protocol was approved by the Ethics Committee of Rafsanjan University of Medical Sciences, Rafsanjan, Iran, in 2019. (IR.RUMS.REC.1397.238).

Funding/Support: The current study was financially supported by Rafsanjan university of medical 
sciences, Rafsanjan, Iran.

Informed Consent: Informed consent was obtained from MS patients before the study.

\section{References}

1. Dobson R, Giovannoni G. Multiple sclerosis-a review. Eur J Neurol. 2019;26(1):27-40. doi: 10.1111/ene.13819. [PubMed: 30300457].

2. Morgen K, Sammer G, Courtney SM, Wolters T, Melchior H, Blecker CR, et al. Evidence for a direct association between cortical atrophy and cognitive impairment in relapsingremitting MS. Neuroimage. 2006;30(3):891-8. doi: 10.1016/j. neuroimage.2005.10.032. [PubMed: 16360321].

3. Ascherio A. Environmental factors in multiple sclerosis. Expert Rev Neurother. 2013;13(12 Suppl):3-9. doi: 10.1586/14737175. 2013.865866. [PubMed: 24289836].

4. Guimarães J, Sá MJ. Cognitive dysfunction in multiple sclerosis. Front Neurol. 2012;3:74. doi: 10.3389/fneur.2012.00074. [PubMed: 22654782].

5. Clemens L, Langdon D. How does cognition relate to employment in multiple sclerosis? A systematic review. Mult Scler Relat Disord. 2018;26:183-91. doi: 10.1016/j.msard.2018.09.018. [PubMed: 30268039].

6. Schiavolin S, Leonardi M, Giovannetti AM, Antozzi C, Brambilla $\mathrm{L}$, Confalonieri $\mathrm{P}$, et al. Factors related to difficulties with employment in patients with multiple sclerosis: a review of 2002-2011 literature. Int J Rehabil Res. 2013;36(2):105-11. doi: 10.1097/MRR.0b013e32835c79ea. [PubMed: 23238667].

7. American Psychiatric Association. Diagnostic and statistical manual of mental disorders (DSM-5®). Arlington, VA: American Psychiatric Pub; 2013.

8. Jongen PJ, Ter Horst AT, Brands AM. Cognitive impairment in multiple sclerosis. Minerva Med. 2012;103(2):73-96. [PubMed: 22513513].

9. Di Filippo M, Portaccio E, Mancini A, Calabresi P. Multiple sclerosis and cognition: synaptic failure and network dysfunction. Nat Rev Neurosci. 2018;19(10):599-609. doi: 10.1038/s41583-018-0053-9. [PubMed: 30158590].

10. Jeannerod M. Mental imagery in the motor context. Neuropsychologia. 1995;33(11):1419-32. doi: 10.1016/00283932(95)00073-c. [PubMed: 8584178].

11. Decety J. The neurophysiological basis of motor imagery. Behav Brain Res. 1996;77(1-2):45-52. doi: 10.1016/01664328(95)00225-1. [PubMed: 8762158].

12. Jeannerod M, Frak V. Mental imaging of motor activity in humans. Curr Opin Neurobiol. 1999;9(6):735-9. doi: 10.1016/s0959-4388(99)00038-0. [PubMed: 10607647].

13. McAvinue LP, Robertson IH. Measuring motor imagery ability: a review. Eur J Cogn Psychol. 2008;20(2):232-51. doi: 10.1080/09541440701394624.

14. Heremans E, D’hooge AM, De Bondt S, Helsen W, Feys P. The relation between cognitive and motor dysfunction and motor imagery ability in patients with multiple sclerosis. Mult Scler. 2012;18(9):1303-9. doi: 10.1177/1352458512437812. [PubMed: 22389414].
15. Tabrizi YM, Zangiabadi N, Mazhari S, Zolala F. The reliability and validity study of the Kinesthetic and Visual Imagery Questionnaire in individuals with multiple sclerosis. Braz J Phys Ther. 2013;17(6):588-92. doi: 10.1590/S141335552012005000124. [PubMed: 24271091].

16. Azin M, Zangiabadi N, Tabrizi YM, Iranmanesh F, Baneshi MR. Deficiency in mental rotation of upper and lower-limbs in patients with multiple sclerosis and its relation with cognitive functions. Acta Med Iran. 2016;54(8):510-7. [PubMed: 27701721].

17. Tabrizi YM, Mazhari S, Nazari MA, Zangiabadi N, Sheibani V, Azarang S. Compromised motor imagery ability in individuals with multiple sclerosis and mild physical disability: an ERP study. Clin Neurol Neurosurg2013;115(9):1738-44. doi: 10.1016/j.clineuro.2013.04.002. [PubMed: 23639730].

18. Tabrizi YM, Mazhari S, Nazari MA, Zangiabadi N, Sheibani V. Abnormalities of motor imagery and relationship with depressive symptoms in mildly disabling relapsing-remitting multiple sclerosis. J Neurol Phys Ther. 2014;38(2):111-8. doi: 10.1097/NPT.0000000000000033. [PubMed: 24531344].

19. Malouin F, Richards CL, Durand A, Doyon J. Reliability of mental chronometry for assessing motor imagery ability after stroke. Arch Phys Med Rehabil. 2008;89(2):311-9. doi: 10.1016/j.apmr.2007.11.006. [PubMed: 18226656].

20. Heremans E, Vercruysse S, Spildooren J, Feys P, Helsen WF, Nieuwboer A. Evaluation of motor imagery ability in neurological patients: a review. Movem Sport Sci. 2013;4:31-8. doi: $10.3917 / \mathrm{sm} .082 .0031$.

21. Macías Islas MÁ, Ciampi E. Assessment and impact of cognitive impairment in multiple sclerosis: an overview. Biomedicines. 2019;7(1):22. doi: 10.3390/biomedicines7010022. [PubMed: 30893874].

22. Heremans E, Feys $P$, Nieuwboer A, Vercruysse $S$ Vandenberghe W, Sharma N, et al. Motor imagery ability in patients with early-and mid-stage Parkinson disease. Neurorehabil Neural Repair. 2011;25(2):168-77. doi: 10.1177/1545968310370750. [PubMed: 21239707].

23. Polman $\mathrm{CH}$, Reingold SC, Banwell B, Clanet M, Cohen JA, Filippi $\mathrm{M}$, et al. Diagnostic criteria for multiple sclerosis: 2010 revisions to the McDonald criteria. Ann Neurol. 2011; 69(2):292-302. doi: 10.1002/ana.22366. [PubMed: 21387374].

24. Oldfield RC. The assessment and analysis of handedness: the Edinburgh inventory. Neuropsychologia. 1971;9(1):97-113. doi: 10.1016/0028-3932(71)90067-4. [PubMed: 5146491].

25. Kurtzke JF. Rating neurologic impairment in multiple sclerosis: an expanded disability status scale (EDSS). Neurology. 1983;33(11):1444-52. doi: 10.1212/wnl.33.11.1444. [PubMed: 6685237]

26. Folstein MF, Folstein SE, McHugh PR. "Mini-mental state". A practical method for grading the cognitive state of patients for the clinician. J Psychiatr Res. 1975;12(3):189-98. doi: 10.1016/0022-3956(75)90026-6. [PubMed: 1202204].

27. Mathiowetz V, Volland G, Kashman N, Weber K. Adult norms for the box and block test of manual dexterity. Am J Occup Ther. 1985;39(6):386-91. doi: 10.5014/ajot.39.6.386. [PubMed: 3160243]. 